\title{
Anesthetic experience of a patient with Ohtahara syndrome -A case report-
}

\author{
Eun Mi Choi ${ }^{1}$, Kyeong Tae $\mathrm{Min}^{2}$, Jin Sun $\mathrm{Cho}^{2}$, and Seung Ho Choi ${ }^{2}$ \\ ${ }^{1}$ Department of Anesthesiology and Pain Medicine, Hallym University College of Medicine, Chuncheon, ${ }^{2}$ Department of \\ Anesthesiology and Pain Medicine, Anesthesia and Pain Research Institute, Yonsei University College of Medicine, Seoul, Korea
}

Ohtahara syndrome (OS) is a rare epileptic encephalopathy that is characterized by an abnormal electroencephalogram (EEG) and intractable seizures in the neonatal and early infantile period. The patient of this reported case was delivered normally at 39 weeks of gestation without any complication. One week after birth, seizures that were refractory to anticonvulsants started with repetitive clustered tonic spasms. The child had no abnormal findings on the initial laboratory investigations. But he was diagnosed with OS according to the frequent tonic spasms, an abnormal EEG pattern of suppression-burst and magnetic resonance imaging of cortical dysplasia. He was planned to undergo an operation for brain lesion. This report describes our experience with the general anesthetic management when we performed craniotomy and right hemispherotomy for a patient with OS. (Korean J Anesthesiol 2011; 60: 124-127)

Key Words: Infantile epileptic syndrome, Ohtahara syndrome, Suppression-burst.

Ohtahara syndrome (OS) is an early infantile epileptic syndrome that was first described in 1976 by Ohtahara et al. [1]. Patients with OS have a characteristic electroencephalogram (EEG), namely, a suppression-burst (SB) pattern during both the sleeping and awake states, as well as frequent tonic spasms [2]. OS is a progressive neurological disorder that involving severe psychomotor retardation and intractable seizures [2]. It has been associated with structural brain malformations or destructive encephalopathy. Moreover, most of patients are refractory to anticonvulsants, so many cases eventually need surgical intervention $[3,4]$. Since the surgical approach is particularly difficult in very young infants [5], reports on the anesthetic management or surgical treatment for OS are rare. So, we report here on the safe use of general anesthesia when performing hemispherotomy in a child suffering with OS.

\section{Case Report}

A 59-day-old, $5.3 \mathrm{~kg}$, male patient presented for general anesthesia for undergoing craniotomy and vertical parasagittal right hemispherotomy. He was normally delivered at 39 weeks of gestation with a birth weight of 3,180 g without any complications or abnormal findings. One week after birth, his seizures started with repetitive clustered tonic spasms for 30

Received: July 12, 2010. Revised: September 13, 2010. Accepted: September 17, 2010.

Corresponding author: Seung Ho Choi, M.D., Department of Anesthesiology and Pain Medicine, Gangnam Severance Hospital, Yonsei University College of Medicine, 146-92, Dogok-dong, Gangnam-gu, Seoul 135-720, Korea. Tel: 82-2-2019-2740, Fax: 82-2-3463-0940, E-mail: csho99@yuhs.ac (C) This is an open-access article distributed under the terms of the Creative Commons Attribution Non-Commercial License (http:// creativecommons.org/licenses/by-nc/3.0/), which permits unrestricted non-commercial use, distribution, and reproduction in any medium, provided the original work is properly cited. 

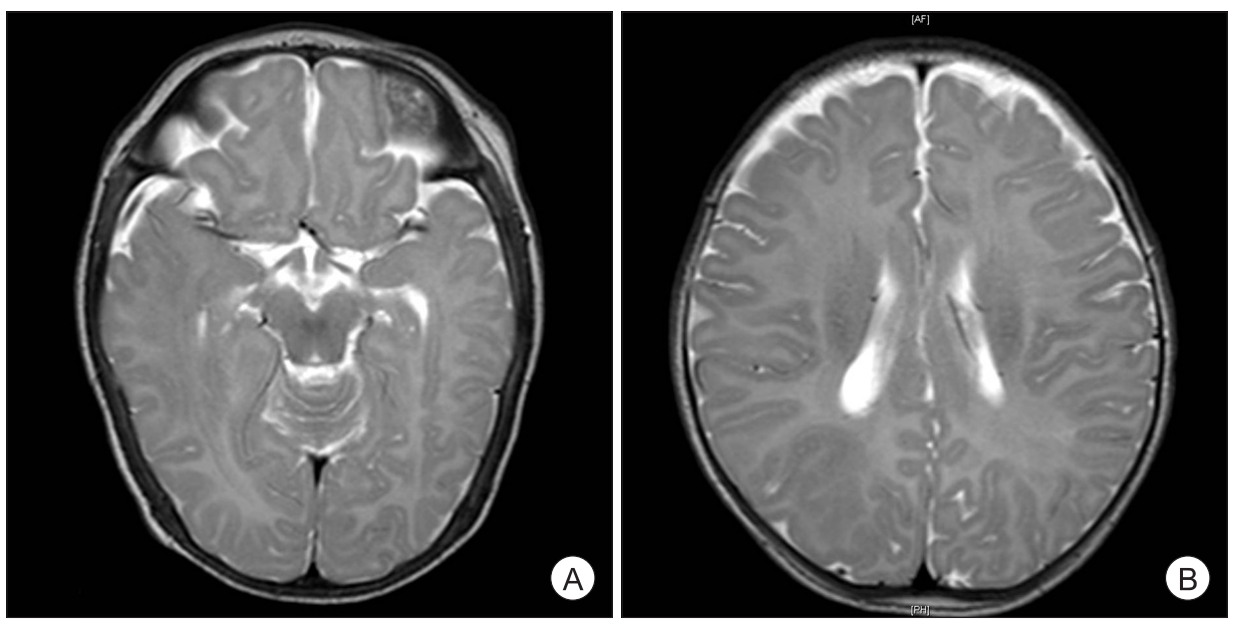

Fig. 1. Magnetic resonance imaging shows a structural abnormality of cortical dysplasia in the right temporal (A) and parietal (B) lobes.

min, at a frequency of 10 clusters a day. He was admitted at an outside hospital, and vigabatrin $250 \mathrm{mg}$ and phenobarbital $20 \mathrm{mg}$ were administered. Yet his seizures were refractory to anticonvulsants, so he was transferred to our hospital at the age of 30 days.

There were no abnormal physical findings and laboratory investigations. The electrocardiogram and chest X-ray were also normal. After admission, he had generalized tonic seizure with right-ward eyeball deviation, blinking and nystagmus at a frequency of $1-3$ a day. Flexor spasms in clusters were also observed for 10 minutes, at a frequency of 7 clusters a day. His magnetic resonance imaging (MRI) showed cortical dysplasia at the right temporal and parietal lobes (Fig. 1). On the positron emission tomography (PET) study, abnormal high uptake for that age was discovered at the right cerebral hemisphere. His video EEG for 2 days demonstrated excessively asynchronous and discontinuous rhythms, the so called suppression-burst patterns, and this was consistent with OS during both the awake and asleep states (Fig. 2). He was diagnosed with OS and right hemispherotomy was planned for treatment.

He was transported to the operating room and the immediate vital signs revealed a blood pressure, pulse rate and oxygen saturation of $83 / 42 \mathrm{mmHg}, 152$ beats/min and $100 \%$, respectively. Before induction, $0.02 \mathrm{mg}$ of glycopyrrolate was injected intravenously. The patient was preoxygenated and general anesthesia was induced with thiopental sodium $25 \mathrm{mg}$ and atracurium $3 \mathrm{mg}$. Mask ventilation was begun with 100\% oxygen and there was no difficulty in ventilation. After confirming full relaxation, the patient's trachea was intubated with a $3.5-\mathrm{mm}$ ID endotracheal tube and volume controlled ventilation was provided with a tidal volume of $45 \mathrm{ml}$. The respiratory rate was adjusted to maintain the end-tidal carbon dioxide $\left(\mathrm{EtCO}_{2}\right)$ at 35-40 mmHg. Anesthesia was maintained with sevoflurane $2.5 \mathrm{vol} \%, \mathrm{FiO}_{2} 0.5$ with air and $2 \mathrm{~L} / \mathrm{min}$ of inspiratory fresh gas

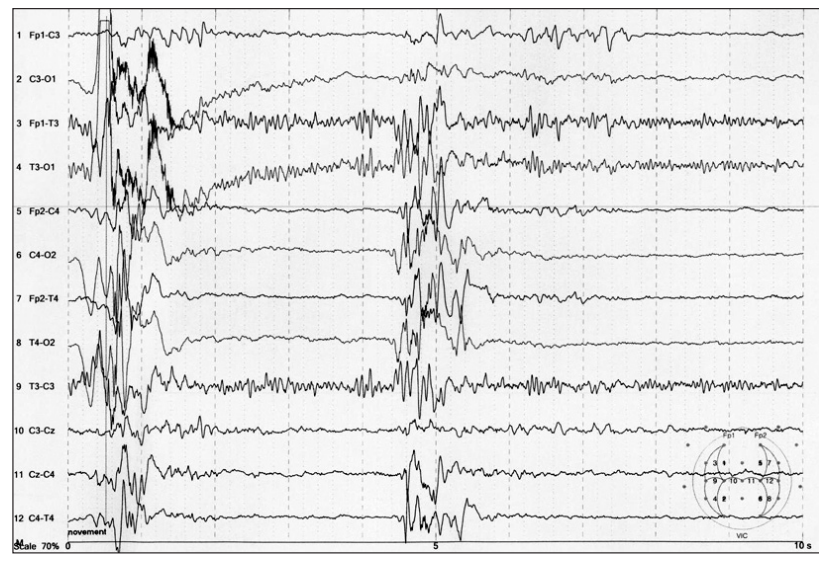

Fig. 2. The electroencephalogram shows excessively asynchronous and discontinuous rhythms, the so called suppression-burst patterns, on the right hemisphere.

flow. A 24-G catheter was placed in the left radial artery for continuous BP monitoring and arterial sampling, and 20-G catheter was inserted into the right femoral vein.

During surgery, the blood pressure, pulse rate and oxygen saturation were stable at $62-74 / 38-51 \mathrm{mmHg}, 127-151$ beats/ min and $99-100 \%$, respectively. The intra-operative arterial blood gas analysis was in the normal range, and the $\mathrm{pH}$, partial pressure of arterial oxygen $\left(\mathrm{PaO}_{2}\right)$ and partial pressure of arterial carbon dioxide $\left(\mathrm{PaCO}_{2}\right)$ was $7.43,173.2 \mathrm{mmHg}$ and 33.1 $\mathrm{mmHg}$, respectively. On completion of surgery, spontaneous respiration was fully restored by manual ventilation. Atropine $0.05 \mathrm{mg}$ and neostigmine $0.1 \mathrm{mg}$ were administrated to reverse the residual muscle relaxation. The endotracheal tube was removed after confirming that the patient was awake and the muscle function had been fully restored. The operation was uneventful. The patient was transported to the post-anesthetic care unit (PACU). The operation time was $6 \mathrm{~h} 45 \mathrm{~min}$, and the 
duration of anesthesia was $7 \mathrm{~h} 55 \mathrm{~min}$. The total amount of fluids administered was $400 \mathrm{ml}$ of $1: 4$ dextrose solution and $500 \mathrm{ml}$ of normal saline, and $200 \mathrm{ml}$ of packed RBCs were also transfused. The total blood loss was $170 \mathrm{ml}$ and the urine output was checked to be $280 \mathrm{ml}$. The preoperative level of hemoglobin was $9.0 \mathrm{~g} / \mathrm{dl}$, and the postoperative level was $10.5 \mathrm{~g} / \mathrm{dl}$.

In the PACU, the patient showed stable vital signs and a regular breathing pattern. His mentality was alert and there was no newly developed neurologic deficit. There were no complications, including hematoma or loss of distal pulsation on the sites of the radial arterial and femoral venous cannulation. He stayed for $50 \mathrm{~min}$ in the PACU and then he was moved to the neurologic intensive care unit. He had no seizure in the first 7 days after surgery and then he was discharged. During the 2 months after surgery, he had no seizure and he has been followed up at our hospital.

\section{Discussion}

OS is a rare epileptic syndrome with a poor prognosis. The characterized seizure pattern of OS is tonic spasms that occur not only when awake, but also during the sleeping state in most cases [6]. It is not difficult to diagnosis OS if we know about patient's EEG finding. The main EEG finding is the SB pattern and this is persistently observed regardless of the circadian cycle. Bursts of high voltage slow waves mixed with spikes that last $1-3 \mathrm{~s}$ alternate with suppression periods that last $3-5 \mathrm{~s}$ at an approximately regular rate. There are also $5-10 \mathrm{~s}$ of burstburst intervals and tonic spasms also appear concomitant with the bursts [6].

The clinical course of OS is progressive with uncontrolled seizure despite of anticonvulsants treatment [7]. Some children evolve into other epileptic encephalopathy such as West syndrome (WS) or Lennox-Gastaut syndrome (LGS) $[8,9]$. Yamatogi and Ohtahara [8] reported that $75 \%$ of the OS cases progress into WS, and 59\% of the cases of WS progress into LGS, with EEG transition from S-B to hypsarrythmia at around 3-6 months of age, and further from hypsarrhythmia to diffuse slow spike-waves at around the age of 1 , respectively.

The etiology of OS is heterogeneous, and organic and structural brain anomalies are commonly associated with OS. Brain imaging often reveals structural abnormalities such as porencephaly, hemimegalencephalopathy, Aicardi syndrome or cerebral atrophy [6]. No inherited case has been reported. In our case, MRI revealed the structural abnormality of cortical dysplasia in the right temporal and parietal lobes.

The efficacy of anticonvulsants medication is limited. Pyridoxal phosphate, valproate, benzodiazepines and steroid, including liposteroid are often tried, but results are disappointing. Vigabatrin has recently been reported to be first drug of choice
[10]. But vigabatrin was not effective for seizure control in our patient, so we decided to perform an operation. Some cases of successful surgical resection in patients with structural brain abnormalities have been reported. One reported patient underwent hemispherectomy for hemimegalencephaly and another underwent resection for focal cortical dysplasia [3,4].

The challenging aspects of anesthetics for patients with this syndrome are managing young age patients, the reduced ability of patients to cooperate or communicate, the possibility of seizure, fixed flexion deformities of all limbs secondary to contractures and a potential effect of polypharmacy [11]. The anticonvulsant medication should be maintained according to the normal medication regimen until the time of surgery and administration should be resumed as soon as possible to prevent perioperative seizure. Rapid sequence induction is recommended since these patients potentially have a full stomach with the risk of aspiration as related with severe mental retardation. It is reasonable to avoid the use of drugs that can produce seizure activity such as ketamine and methohexital. Although most inhaled anesthetics have been reported to produce seizure, isoflurane and desflurane are reported to be relatively safe and they generally produce dose-dependent depression of EEG activity. Although sevoflurane may be epileptogenic, the clinical significance of this is uncertain. We used sevoflurane and no seizure activity was observed during induction. Without EEG monitoring during anesthesia, we were unaware of any seizure activity during general anesthesia, and especially with neuromuscular blockade. But the bispectral index (BIS) has been shown to correlate with numerous EEG conditions [12] and this can be useful in monitoring cerebral activity during surgery. The potential effect of anticonvulsant is another problem that needs to be considered. The patients receiving chronic anticonvulsant therapy may exhibit resistance to the neuromuscular blocking effects of various muscle relaxants [13]. The patient should be fully awake to avoid the risks of regurgitation and aspiration, and a pain free recovery should be planned to reduce cerebral excitation on emergence from anesthesia $[11,14]$.

A review of the medical literature revealed two cases related to general anesthesia for patients with OS. One case was a 15-year-old male with acute testicular torsion [14] and the other case was a 10-year-old male with femur fracture [11]. In their cases, anesthesia was induced with rapid sequence induction and this was maintained with sevoflurane. Atracurium and suxamethonium were used for neuromuscular relaxation: both the patients showed a normal response to neuromuscular blocking and the administered opiate drugs. No seizure attacks occurred during anesthesia.

In conclusion, OS can be easily diagnosed by the characteristic EEG and seizure pattern. For OS patients, the plan for 
anesthesia should be made with considering the possibility of seizure and a potential effect of polypharmacy. We also recommend BIS monitoring during anesthesia because this can be useful to monitor cerebral activity.

\section{References}

1. Ohtahara S, Ishida T, Oka E, Yamatogi Y, Inoue H. On the specific age-dependent epileptic syndrome: the early-infantile epileptic encephalopathy with suppression-burst. No To Hattatsu 1976; 8: 270-80.

2. Clarke M, Gill J, Noronha M, McKinlay I. Early infantile epileptic encephalopathy with suppression burst: Ohtahara syndrome. Dev Med Child Neurol 1987; 29: 520-8.

3. Pedespan JM, Loiseau H, Vital A, Marchal C, Fontan D, Rougier A. Surgical treatment of an early epileptic encephalopathy with suppression-bursts and focal cortical dysplasia. Epilepsia 1995; 36: $37-40$.

4. Komaki H, Sugai K, Maehara T, Shimizu H. Surgical treatment of early-infantile epileptic encephalopathy with suppression-bursts associated with focal cortical dysplasia. Brain Dev 2001; 23: 727-31.

5. Hmaimess G, Raftopoulos C, Kadhim H, Nassogne MC, Ghariani $\mathrm{S}$, de Tourtchaninoff M, et al. Impact of early hemispherotomy in a case of Ohtahara syndrome with left parieto-occipital megalencephaly. Seizure 2005; 14: 439-42.

6. Yamatogi Y, Ohtahara S. Early-infantile epileptic encephalopathy with suppression-bursts, Ohtahara syndrome; its overview referring to our 16 cases. Brain Dev 2002; 24: 13-23.

7. van Rijckevorsel K. Cognitive problems related to epilepsy syndromes, especially malignant epilepsies. Seizure 2006; 15: 227-34.

8. Yamatogi Y, Ohtahara S. Age-dependent epileptic encephalopathy: a longitudinal study. Folia Psychiatr Neurol Jpn 1981; 35: 321-32.

9. Ohtsuka Y, Ogino T, Murakami N, Mimaki N, Kobayashi K, Ohtahara S. Developmental aspects of epilepsy with special reference to agedependent epileptic encephalopathy. Jpn J Psychiatry Neurol 1986; 40: 307-13.

10. Dulac O. Epileptic encephalopathy. Epilepsia 2001; 42 Suppl 3: 23-6.

11. Bruton J, Crowe S. Combined general and regional anesthesia in a child with Ohtahara syndrome. Paediatr Anaesth 2008; 18: 1111-2.

12. Dahaba AA, Liu DW, Metzler H. Bispectral index (BIS) monitoring of acute encephalitis with refractory, repetitive partial seizures (AERRPS). Minerva Anestesiol 2010; 76: 298-301.

13. Tempelhoff R, Modica PA, Jellish WS, Spitznagel EL. Resistance to atracurium-induced neuromuscular blockade in patients with intractable seizure disorders treated with anticonvulsants. Anesth Analg 1990; 71: 665-9.

14. Boyce H, Satya-Krishna R. Anaesthesia in an adolescent with Ohtahara syndrome. Anaesthesia 2003; 58: 1029. 